\title{
Articulatory Strategies for Place Contrasts of Unreleased Final Stops on Preceding Vowels: Evidence from Ultrasound Imaging
}

\author{
Suki Yiu, Diana Archangeli, Jonathan Yip \\ University of Amsterdam, University of Arizona, University of Hong Kong
}

\section{Introduction}

Unreleased final stops are susceptible to reduction, rendering coarticulatory information on the preceding vowel important to signal place contrasts of these postvocalic stops. This study has employed ultrasound imaging to examine the lingual properties of the place of articulation of unreleased final stops on the preceding vowels.

The gestural coordination of vowel-consonant sequences of monosyllabic words in Cantonese represents a testing case for having preserved phonemic contrasts of six unreleased final stops in a range of vowel contexts. In Cantonese, unreleased final stops $/ \mathrm{p} /, / \mathrm{t} /, / \mathrm{k} /, / \mathrm{m} /, / \mathrm{n} /$ and $/ \mathrm{y} /$ can be preceded by vowels such as $/ \mathrm{i} /, / \mathrm{e} /, / \mathrm{a} /, / \mathrm{o} /$ and $/ \mathrm{u} /$. Khouw and Ciocca's (2006) acoustic study found that unreleased final stops in Cantonese use consonant-to-vowel coarticulation to strengthen place contrasts, providing us an acoustic ground for verification.

Three hypotheses are presented as follows.

(1) Hypothesis 1. Minimal coarticulation: Place contrasts are only present at vowel offsets.

Hypothesis 2. Gradual coarticulation: Place contrasts are present at vowel midpoints.

Hypothesis 3. Vowel $\leftarrow$ consonant phonologisation: Place contrasts are present throughout the vowel.

\section{Methods}

2.1 Participants Ten native Cantonese participants were recruited at the University of Hong Kong. The number of participants was balanced by gender. They were asked to fill in a language background questionnaire after the experiment, in particular to report on other languages that they also spoke and languages that were used with different groups in different contexts.

2.2 Stimuli The unreleased final stops selected in this study are $/ \mathrm{t} /, / \mathrm{k} /, / \mathrm{n} /$ and $/ \mathrm{n} /$, and the vowels involved are $/ \mathrm{i} /, / \mathrm{e} /, / \mathrm{a} /, / \mathrm{\rho} / \mathrm{and} / \mathrm{u} /$. These vowel-consonant sequences were either onsetless or combined with a relatively lingual neutral onset to minimize any onset incurred coarticulatory effects on the following vowel. The target sequences were then placed in two carrier frames ending with a sentence-final particle either /a/ or /po/. 4000 tokens of dada were recorded from 5 vowels x 4 consonants x 2 examples x 2 carrier frames $\mathrm{x} 5$ iterations $\mathrm{x} 10$ participants.

2.3 Data collection Participants were instructed to read the randomized sentences naturally from a computer screen, where one sentence was displayed each time. The whole list was repeated five times. The

\footnotetext{
* The authors sincerely thank Stephen Matthews for various discussions, Eric Liu for involving in data processing, and the ten subjects for participating in the study. This project has benefited from valuable discussion with and feedback by, in particular, Jie Zhang, Feng-fan Hsieh, Bryan Gick, John Kingston, Douglas Whalen, Ryan Bennett, Alexei Kochetov, and Jason Shaw. The authors also thank the organisers, reviewers and audience of AMP 2020 (UC Santa Cruz), and ASA 172 (Hawai'i). This project is funded by a University Research Council grant (Hong Kong) to the first author.
}

(C) 2021 Suki Yiu, Diana Archangeli, and Jonathan Yip

Proceedings of AMP 2020 
data was recorded in a quiet room of the Department of Linguistics at the University of Hong Kong. Acoustic data was recorded by using an earset microphone at a sampling rate of $44.1 \mathrm{kHz}$ with Praat (Boersma \& Weenick, 2016). While acoustic measures are hardly available for the comparison of the amount of coarticulatory information on the preceding vowels with the closure target of consonants, in Articulatory Phonology (Goldstein \& Fowler, 2003; Goldstein, Byrd, \& Saltzman, 2006; Goldstein, Nam, Saltzman, \& Chitoran, 2009), articulatory events are typically planned to facilitate synchronous movements of the articulators involved in speech. Articulatory data was thus also collected using an ultrasound scanner with a MC4 2-4 MHz micro-convex ultrasound transducer. Continuous ultrasound video was captured with Fraps (Beepa, 2004-2015) at $60 \mathrm{fps}$.

2.4 Data analysis The data was then segmented at vowel onset, midpoint and offset positions with Praat (Boersma \& Weenick, 2016). The tongue contours from the ultrasound video were extracted using EdgeTrak (Li, Kambhamettu, \& Stone, 2005) at vowel onset, midpoint, and offset (Recasens \& Rodríguez, 2015). A Smoothing Spline ANOVA (SS ANOVA) (Davidson, 2006) of vowel contours was performed with $\mathrm{R}$ (R Core Team, 2018) to examine the place contrasts on different vowels at those three time points. Linear mixed-effects regression (LMER) analyses were then run to the distance between the origin and the point on each spline closest to the angles identified for coronals and dorsals from respective splines at the vowel offset in order to inspect whether the progress of gestural achievement is significant over time. The contrast between high and non-high vowels in terms of vowel qualities could be very different between coronals and dorsals, which will be discussed more thoroughly in the next section.

\section{Results}

The SS ANOVA plots of one participant is adopted in Figure 1 to illustrate the gestural progression from vowel onset, midpoint, and offset to the unreleased final stops. The tongue contours for [pet] 'no' and [pek] 'north' are represented by the purple and lime splines respectively. As we can see, the supposedly same vowel [e] in [pet] and [pek] looks more distinct in the two contexts as the articulation progresses towards the vowel offset. This indicates that the closer to the vowel offset, the more articulatory properties of the unreleased final stops $[\mathrm{t}]$ and $[\mathrm{k}]$ are available on the preceding vowel $[\mathrm{k}]$.

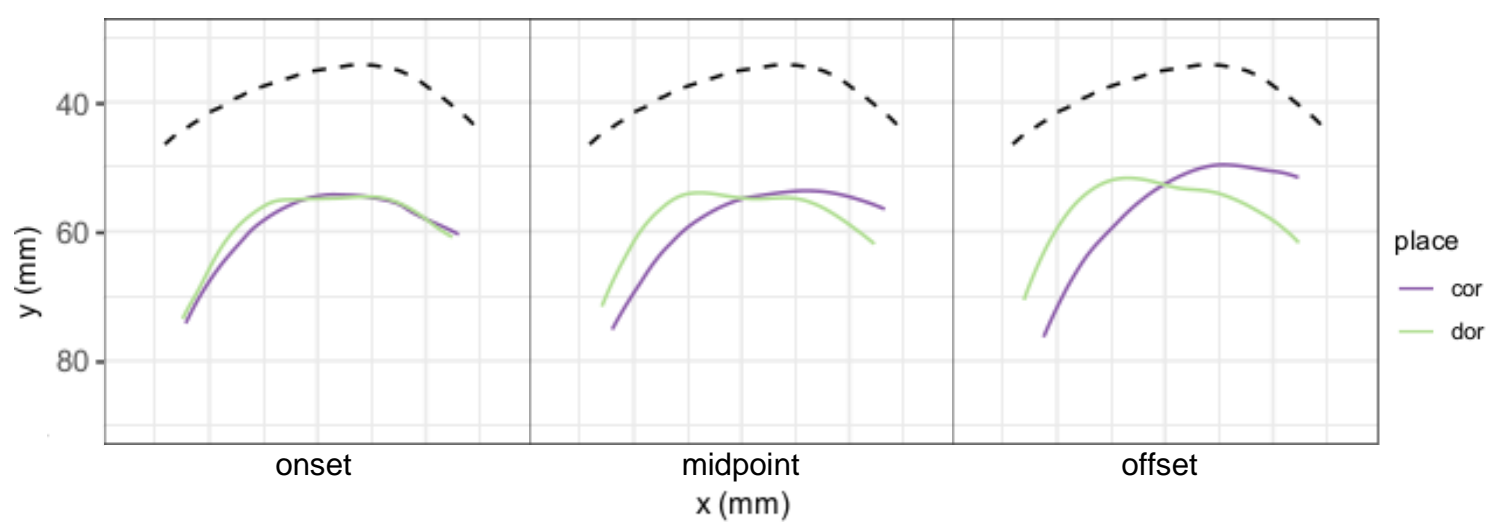

Figure 1. SS ANOVA plots of [pet] 'no' vs. [pek] 'north' at vowel onset, midpoint and offset positions. Splines for [pet] 'no' and [pek] 'north' are in purple and lime respectively.

When comparing the high and non-high vowels preceding coronals and dorsals at vowel onset, midpoint and offset, preliminary results display two coarticulatory patterns depending on the vowel height, which will be discussed in sections 3.1 and 3.2. 
3.1 Non-high vowels Figure 2 compares high [i], [u] and non-high [e], [a], [0] vowels preceding coronals [t], [n] and dorsals $[\mathrm{k}],[\mathrm{y}]$ at vowel onset, midpoint and offset when preceding coronals (top panel) and dorsals (bottom panel). The higher the boxes in the boxplots, the farther the point of a spline from the origin, the better the gesture is achieved.

For non-high vowels, gestural changes are highly significant from vowel onset to midpoint for coronals (est $=79.73, t=8.713, p<.001$ ), from vowel midpoint to offset for both coronals (est $=76.84, t=$ $4.523, p<.001)$ and for dorsals (est $=71.02, t=11.705, p<.001$ ), and from vowel onset to offset for both coronals (est $=79.73, t=4.191, p<.001)$ and dorsals (est $=71.61, t=9.775, p<.001)$, indicating gradual consonant-to-vowel coarticulation.

3.2 High vowels For high vowels, however, gestural changes for coronals are insignificant except from vowel onset to midpoint (est $=85.65, t=2.683, p<.01)$. Gestural changes for dorsals are insignificant except from vowel onset to midpoint (est $=71.53, t=3.411, p<.001)$ and from vowel onset to vowel offset (est $=75.53, t=2.091, p<.05$ ). This can be interpreted as gestural transition being completed by vowel midpoint for both coronals and dorsals, indicating phonologisation of place information of the unreleased final stop on the preceding vowel.

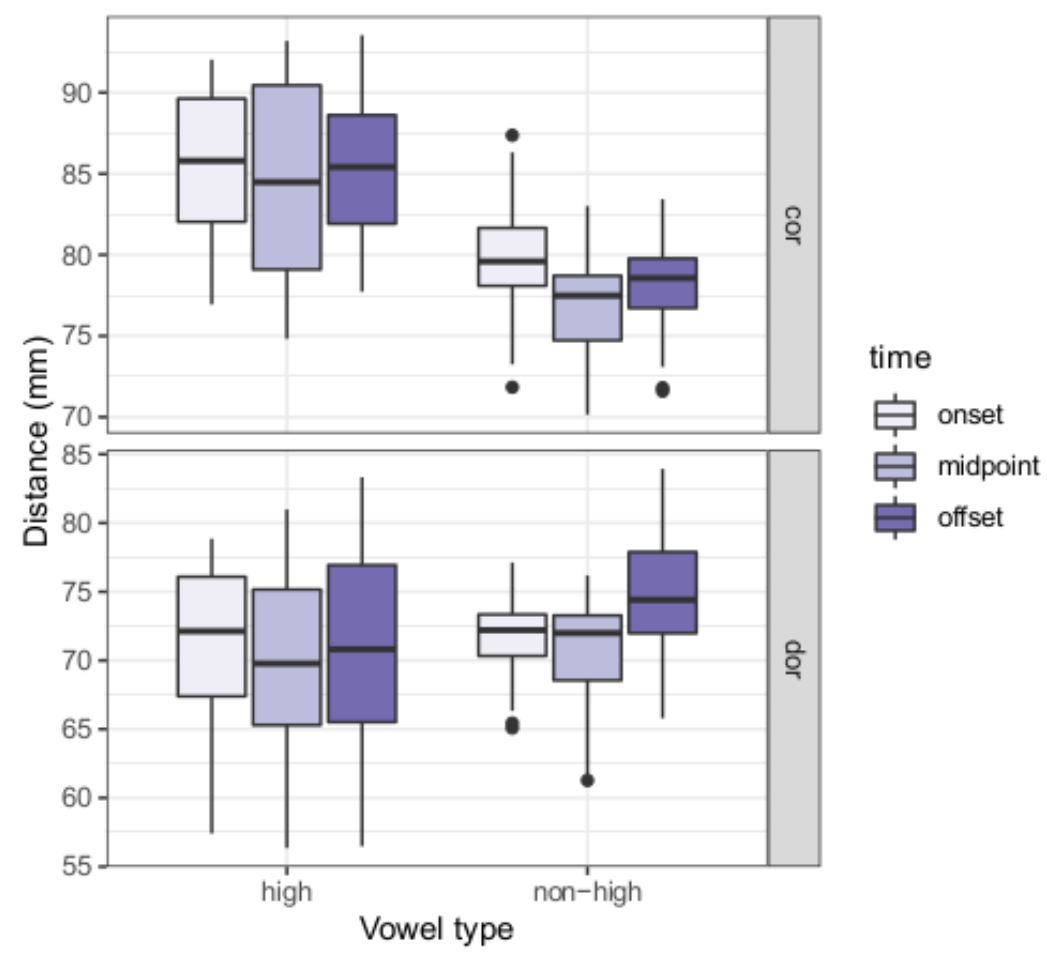

Figure 2. Progress of gestural achievement for high vs. non-high vowels at vowel onset (light purple), midpoint (purple) and offset (dark purple). Vowels preceding coronal stops are shown in the top panel, while vowels preceding dorsal stops are in the bottom panel.

\section{Discussion and conclusions}

Based on the preliminary results, non-high vowels are undergoing gradual anticipatory coarticulation, where significant gestural changes are mainly reported from vowel midpoint to offset and from vowel onset 
to offset. This confirms Hypothesis 2 for non-high vowels. In contrast, gestural changes for high vowels are mainly reported from vowel onset to midpoint, indicating early lingual raising from vowel onset to midpoint into final stop constrictions, which is different from approaching to a gestural target over the entire course as for non-high vowels, and thus closer to the stage of phonologisation. This confirms Hypothesis 3 for high vowels. The results of this paper agree with an earlier acoustic study (Khouw \& Ciocca, 2006), which has also adopted Cantonese as a testing case, that unreleased final stops use consonant-to-vowel coarticulation to strengthen place contrasts.

This paper has presented articulation data via ultrasound imaging for the coarticulation of unreleased final stops and their preceding vowels, in particular, the amount of lingual coarticulation properties of those unreleased final stops on the preceding vowels. The results show that coarticulatory patterns depend on vowel height, that is, non-high vowels are undergoing gradual coarticulation whereas high vowels are phonologising the lingual properties of the unreleased final stops on the preceding vowels.

Further analyses shall take interspeaker variation into account to see whether there might be clearer talkers than others, which might be relevant for the analysis of non-high vowels, given that the amount of coarticulatory information on the vowel is dependent on the extent to which a fully articulated gestural target is available at the end for the anticipatory coarticulation to take place.

In relation to the availability of a fully articulated gestural target, Yip (2015) found systematic patterns of reduction of the anterior or posterior constrictions for $/ \mathrm{t} / \mathrm{and} / \mathrm{k} /$ according to the place of the following consonant of the word. The lingual gestures of pre-labial stops tended to be more reduced than the same gestures in the other contexts. We then expect the target $/ \mathrm{t} /$ and $/ \mathrm{k} /$ might be less fully articulated when put in one of the carrier frames where the unreleased final stops precede a syllable beginning with a labial of the sentence final particle /po/.

Further analyses can also check whether stops and nasals behave differently. Law, Fung, and Bauer (2001) reported that words ending with oral stops are more prone to being misperceived and misproduced than words ending with nasals, which may due to less acoustic information available for oral stops for both speakers and listeners. Therefore, if there may be a difference, we expect the non-high vowels preceding nasals to carry more coarticulatory information than those preceding oral stops.

In addition to the articulation data, acoustics analysis can be performed to examine the quality of coarticulated non-high vowels and coarticulated/phonologised high vowels.

\section{References}

Beepa (2004-2015). Fraps [computer program]. Version 3.5.99. http://www.fraps.com.

Boersma, Paul, \& Weenink, David. (2016). Praat: doing phonetics by computer [computer program]. Version 5.4.06. http://www.praat.org.

Davidson, Lisa. (2006). Comparing tongue shapes from ultrasound imaging using smoothing spline analysis of variance. Journal of the Acoustical Society of America 120 (1), 407-415.

Goldstein, Louis, Byrd, Dani, \& Saltzman, Elliot. (2006). The role of vocal tract gestural action units in understanding the evolution of phonology. In Arbib, Michael (Ed.), Action to Language via the Mirror Neuron System. Cambridge University Press: 215-249.

Goldstein, Louis, \& Fowler, Carol. (2003). Articulatory Phonology: A phonology for public language use. In: Schiller, Neils, Meyer, Antje (Eds.), Phonetics and Phonology in Language Comprehension and Production. Mouton de Gruyter: 159-207.

Goldstein, Louis, Nam, Hosung, Saltzman, Elliot, \& Chitoran, Ioana. (2009). Coupled oscillator planning model of speech timing and syllable structure. In Fant, Gunnar, Fujisaki, Hiroya, Shen, Jiaxuen (Eds.), Frontiers in Phonetics and Speech Science. The Commercial Press: 239-250.

Khouw, Edward, \& Ciocca, Valter. (2006). An acoustic and perceptual study of final stops produced by profundly hearing impaired adolescents. Journal of Speech, Language, and Hearing Research 49, 172-185.

Law, Sam Po, Fung, Roxanna, \& Bauer, Robert. (2001). Perception and production of Cantonese consonant endings. Asia Pacific Journal of Speech, Language and Hearing 6, 179-195.

Li, Min, Kambhamettu, Chandra, \& Stone, Maureen. (2005) Automatic contour tracking in ultrasound images. Clinical Linguistics and Phonetics 19(6-7), 545-554.

R Core Team. (2018). R: A language and environment for statistical computing. R Foundation for Statistical Computing, Vienna, Austria. URL https://www.R-project.org/. Version 3.4.3.

Recasens, Daniel, \& Rodríguez, Clara. (2015). An investigation of lingual coarticulation resistance using ultrasound. In Abstract book of Ultrafest VII. Hong Kong, The University of Hong Kong. 
Yiu, et al.

Articulatory Strategies for Place Contrasts of Unreleased Final Stops on Preceding Vowels

Yip, Jonathan. (2015). Gestural reduction of Hong Kong Cantonese syllable-final oral stops. In the Scottish Consortium for ICPhS 2015 (Ed.), Proceedings of the 18th International Congress of Phonetic Sciences. 